\title{
Histopathologic Patterns of Recurrent Choroidal Melanoma Following I-125 Plaque Brachytherapy
}

\author{
Jose J. Echegaray ${ }^{\mathrm{a}}$ Thomas Plesec $^{\mathrm{b}}$ Claudine Bellerive $^{\mathrm{a}}$ Arun D. Singh ${ }^{\mathrm{a}}$ \\ ${ }^{a}$ Department of Ophthalmic Oncology, Cole Eye Institute, Cleveland Clinic Foundation, Cleveland, OH, USA; \\ ${ }^{b}$ Department of Anatomic Pathology, Cleveland Clinic Foundation, Cleveland, $\mathrm{OH}$, USA
}

\section{Keywords}

Choroidal melanoma $\cdot$ I-125 plaque

brachytherapy · Local tumor recurrence · Tumor

radioresistance $\cdot$ Immunohistochemistry

\begin{abstract}
Purpose: Histologic correlation of clinical patterns of recurrent choroidal melanoma following I-125 plaque brachytherapy was performed to identify pathologic mechanisms of recurrence. Methods: We reviewed 7 cases of recurrent choroidal melanoma following I-125 plaque brachytherapy managed with enucleation. Clinical characteristics included tumor dimensions, radiation dose, time to local recurrence, and clinical pattern of recurrence. Histopathology (hematoxylin and eosin and periodic acid - Schiff) and immunohistochemistry (Ki-67, CD-163, HMB45, and SOX10) were performed. Results: Mean follow-up time and time to local recurrence were 42 and 21 months after brachytherapy, respectively. Tumor recurrences were described clinically as marginal in 43\%, diffuse in 29\%, and extraocular extension (EOE) in $29 \%$. Eighty-six percent were classified as mixed cell type and $14 \%$ were epithelioid type. Tumor zonation (histologic demarcation between zones of recurrent and nonre-
\end{abstract}

(c) 2019 S. Karger AG, Basel

E-Mail karger@karger.com

www.karger.com/oop current tumor cells by immunohistochemistry) was present in marginal and EOE cases $(n=6)$ and absent in the diffuse cases $(n=2)$. Ki-67 proliferative index was higher in marginal and EOE recurrences, while diffuse cases showed uniform Ki-67 staining. CD-163 staining was found to be greater in nonrecurrent tumor. HMB45 correlated with SOX10 with a greater staining in recurrent tumor. Conclusion: Our observations provide a correlation between histopathologic and clinical patterns of local recurrence of choroidal melanoma after brachytherapy.

(c) 2019 S. Karger AG, Basel

\section{Introduction}

The main therapeutic goal of episcleral plaque brachytherapy for choroidal melanoma is to achieve local tumor control. Local tumor recurrences are associated with a higher risk for metastasis [1]. Our group has previously reported a 5.6\% rate of local tumor recurrence following a median time of 18 months since episcleral brachytherapy with a median time of follow-up of 47 months [2]. Various clinical patterns of recurrence were presented in the reported 21 cases that include marginal, central, dif- 
fuse, ring, or extraocular extension (EOE). Previous reports have suggested similar clinical patterns of recurrence $[1,3]$.

Understanding the pathologic mechanisms of local tumor recurrence after plaque brachytherapy is crucial to improving current treatment strategies and developing novel treatment strategies. However, the published data describing histopathologic patterns of local recurrence of choroidal melanoma after brachytherapy is limited. In a descriptive pathologic study of 59 enucleated eyes with melanoma after plaque brachytherapy, Shields et al. [4] included 30 cases that were enucleated for tumor growth. Their study suggested that tumor regrowth developed in horizontal lateral dimensions more commonly than in thickness with evidence of distinct transition of malignant tumor cells near the tumor margin in 3 eyes. The Collaborative Ocular Melanoma Study (COMS) report No. 6 also provided a histopathologic description of enucleated eyes using light microscopy and found lower mitotic activity in tumors irradiated prior to enucleation [5]. The authors were unable to histologically confirm tumor recurrence in $40 \%$ of cases. In a later COMS report, Avery et al. [6] compared histopathologic findings of eyes with choroidal melanoma treated with primary enucleation with eyes enucleated secondarily after I-125 plaque brachytherapy, including 42 eyes with local recurrence. When compared to tumors that underwent primary enucleation, those treated with secondary enucleation had overall less mitotic activity. In addition, local recurrence could not be confirmed in 17 of 42 eyes with standard hematoxylin and eosin (H\&E). Additionally, one of the pitfalls of the COMS study histopathologic analysis was their description of areas of presumed viable tumor cells in cases of secondary enucleation due to local recurrence [6]. There was no correlation of these areas with immunohistochemistry (IHC) characterization or proliferation indices. Therefore, the histopathologic descriptions from the COMS suggest that light microscopy with standard H\&E may not be sufficient to identify recurrent choroidal melanoma following plaque brachytherapy. Hence, the authors could not identify clinicopathologic patterns of local recurrence.

We used IHC to identify zonal distribution of recurrent tumor for detailed analysis. Correlation with clinical patterns of recurrence was also performed. We identified distinct clinicopathologic patterns of local recurrence of choroidal melanoma following episcleral brachytherapy. Our study also proposes potential preventive strategies for each type of recurrence.

Histopathologic Patterns of Recurrent Choroidal Melanoma

\section{Methods}

We obtained approval from the Institutional Review Board of the Cleveland Clinic. We had previously reported 21 cases of iris, ciliary body, and choroidal melanoma that had local recurrence after plaque brachytherapy and described their clinical patterns [2]. In our present study, we performed a retrospective study of those cases of recurrent choroidal melanoma that were managed with enucleation and had histopathologic analysis $(n=7)$. Clinical characteristics include tumor dimensions, dose to tumor apex, echographic confirmation of plaque placement, adjuvant transpupillary thermotherapy (TTT), time to local recurrence, and clinical type of recurrence. The latter included marginal, EOE, and diffuse types, as previously published by our group. Light microscopy using H\&E and periodic acid - Schiff stains was performed to determine tumor mitotic rates, tumor cellular composition in percentages of melanocytes, hemorrhagic component, macrophages, and fibrotic areas. In addition, each tumor was classified according to its predominant cell type: spindle cell, epithelioid, or mixed type. As in the COMS trial study, when greater than half of the tumor was composed of larger, round cells with abundant cytoplasm and showing poor cohesiveness, the tumor was classified as epithelioid type [5]. If half of the tumor was composed of epithelioid cells, then it was considered mixed cell type [5].

We performed IHC for differentiation between recurrent tumor and nonrecurrent tumor using Ki-67, CD-163, HMB45, and SOX10. We chose Ki-67 to measure cellular proliferation, reported as a percentage index. CD-163 was used as a cytoplasmic marker for macrophages. To identify neoplastic melanocytes, we chose HMB45 and SOX10. HMB45 is a cytoplasmic marker for melanocytic proliferation, and SOX10 is a nuclear marker for a neural crest transcription factor in malignant melanoma. We also performed IHC to identify the presence of tumor zonation, defined as the histologic demarcation between zones of presumed recurrent and nonrecurrent tumor. Previous histopathologic descriptions from the COMS trial referred to intratumoral areas of "presumed viable tumor cells," which represented suspected nonnecrotic neoplastic melanocytes [6]. We labeled any presumed viable tumor cells as "recurrent tumor" and any areas of necrotic tumor cells as "nonrecurrent tumor" in correlation with each respective IHC stain. Each sample was scored by 2 of the authors (J.J.E. and T.P.) using increasing degrees of staining: $0=$ negative, $+1=$ low, $+2=$ moderate, and $+3=$ high .

\section{Results}

Seven cases with a mean follow-up time of 42 months since episcleral plaque brachytherapy were included. Mean tumor largest basal diameter and tumor height were 13.8 and $4.8 \mathrm{~mm}$, respectively. Mean apical dose was 83 Gy. Echographic confirmation of tumor margin coverage was done in 3 cases (43\%). Adjuvant TTT was done in 2 cases (29\%). Average time to local tumor recurrence was 21 months after plaque treatment. The clinical variants of recurrence were described as marginal in $43 \%$, dif-

Ocul Oncol Pathol 2020;6:50-57 
Table 1. Clinical characteristics of recurrent choroidal melanoma following I-125 plaque brachytherapy

\begin{tabular}{lc}
\hline Number of patients & $n=7$ \\
\hline Age at presentation, years, mean (range) & $72.9(68-79)$ \\
Follow-up time, months, mean (range) & $42(10-119)$ \\
Tumor dimensions, mm, mean (range) & \\
$\quad$ LBD & $13.8(5-20)$ \\
$\quad$ Height & $4.8(1.5-8)$ \\
Distance to fovea, mm, mean (range) & $3.1(1-7)$ \\
Distance to disc, mm, mean (range) & $2.8(0-6)$ \\
Dose to tumor apex, Gy, mean (range) & $83.2(76.1-94.8)$ \\
Adjuvant TTT, $n$ (\%) & $2(29)$ \\
Echographic confirmation of plaque & $3(43)$ \\
$\quad$ placement, $n$ (\%) & \\
Time to recurrence after plaque, months, & $21(7-59)$ \\
$\quad$ mean (range) & $3(43)$ \\
Clinical pattern of recurrence, $n$ (\%) & $2(29)$ \\
$\quad$ Margin & $2(29)$ \\
EOE & $3(43)$ \\
Diffuse, central & $3(43)$ \\
Liver metastasis &
\end{tabular}

TTT, transpupillary thermotherapy; EOE, extraocular extension.

fuse/central in 29\%, and EOE in 29\%. Marginal and diffuse/central recurrences were detected with indirect ophthalmoscopy, while the EOE variant was diagnosed with ultrasound B-scan. Forty-three percent of cases had liver metastases (Table 1).

Three histopathologic types of recurrences were identified: marginal $(n=3)$, EOE $(n=2)$, and diffuse $(n=2)$. Distinct tumor zonation between recurrent and nonrecurrent tumor, not identifiable with $\mathrm{H} \& \mathrm{E}$ and periodic acid - Schiff stains, was distinctively visible by IHC in 5 cases (marginal $[n=3]$ and EOE $[n=2]$ ) and absent in 2 diffuse cases (Fig. 1-3). Two cases clinically described as diffuse and central recurrences shared similar histologic features and were therefore grouped together within the diffuse histopathologic type. Six cases (86\%), including marginal and EOE cases, were classified as mixed cell type tumors. One diffuse case (14\%) was classified as epithelioid type. The mitotic rate per 40 high-power fields ranged between 0 and 3. Tumor cell composition differed between diffuse recurrence cases and marginal and EOE cases. Diffusely recurrent tumors had a histopathologic composition of $40-60 \%$ melanocytic cells, $30-40 \%$ hemorrhagic areas, and 10-20\% macrophages. Tumors with marginal and EOE recurrences were composed of $90-95 \%$ melanocytic cells,
$0-5 \%$ macrophages, and $0-10 \%$ fibrotic areas. In addition, those cases with EOE showed transscleral emissary channel microinvasion (Table 2).

IHC showed that in all marginal and EOE cases $(n=5)$ there was a higher Ki-67 proliferation index in clinically observed areas of tumor recurrence than in areas that appeared clinically stable after episcleral brachytherapy. Two diffuse recurrence cases showed uniform degree of Ki-67 staining throughout the tumor. CD-163 macrophage markers showed mildly higher degree of staining in the non-recurrent portions of the tumors $(n=5)$. HMB45 staining correlated with SOX10 with a higher degree of staining in recurrent areas in marginal and EOE types. The diffuse recurrences showed uniformly high degree of HMB45 and SOX10 staining throughout the tumor, which correlate with the uniform Ki-67 distribution. This represents absence of tumor zonation by IHC, in contrast with the marginal and EOE cases which show zonation of recurrent tumor (Table 3).

\section{Discussion}

Aided by IHC we could discern 3 histopathologic patterns of local tumor recurrence of choroidal melanoma after I-125 plaque brachytherapy: marginal, EOE, and diffuse. The marginal and EOE recurrences correlate with a mixed cell tumor type, show a relatively higher proportion of melanocytic cell composition, and show a recurrent tumor zonation distribution with IHC (Fig. 1, 2 ). This is evident by the correlation of higher degrees of Ki-67, HMB45, and SOX10 staining in the recurrent portions of the tumors. In a collaborative work by the Ocular Oncology Group (EORTC), Ki-67 activity (and PCNA) was significantly higher in melanomas that were recurrent or not irradiated at all than nonrecurrent melanomas (enucleated for neovascular complications) [7]. Higher degree of CD-163 staining in the nonrecurrent areas may represent a more prominent histiocytic cell component in areas that are clinically stable after brachytherapy as part of the postradiation inflammation or debris removal. This may be consistent with reported findings from the COMS trial of eyes treated with primary enucleation, in which a higher degree of macrophages correlated with areas of necrotic tumor cells [5]. Two cases that had been described clinically as diffuse and central recurrences showed a lower proportion of melanocytic cell composition and lacked zonation differences between recurrent and nonrecurrent tumor evident by uniform Ki-67, HMB45, and SOX10 staining (Fig. 3). 

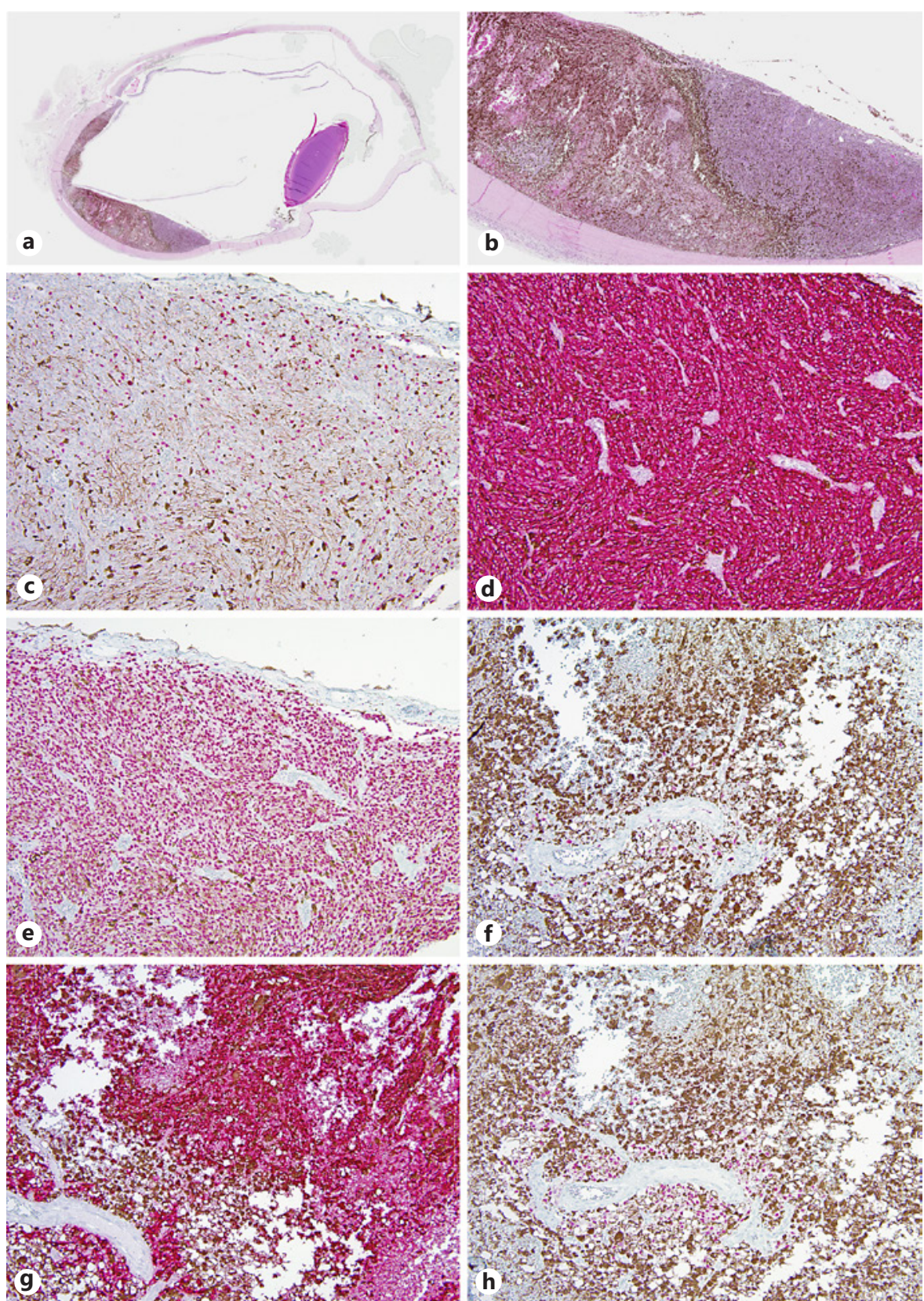

Fig. 1. Marginal recurrence of choroidal melanoma at low (a) and high (b) magnification with H\&E stain. Tumor zonation is illustrated with dense staining of recurrent tumor with Ki-67 (c), HMB45 (d), and SOX10 (e), while mild staining is observed in nonrecurrent tumor with Ki-67 (f), HMB45 (g), and SOX10 (h). Macrophage staining with CD-163 also shows zonation with milder degree of staining in recurrent tumor (i) than in nonrecurrent tumor (j).
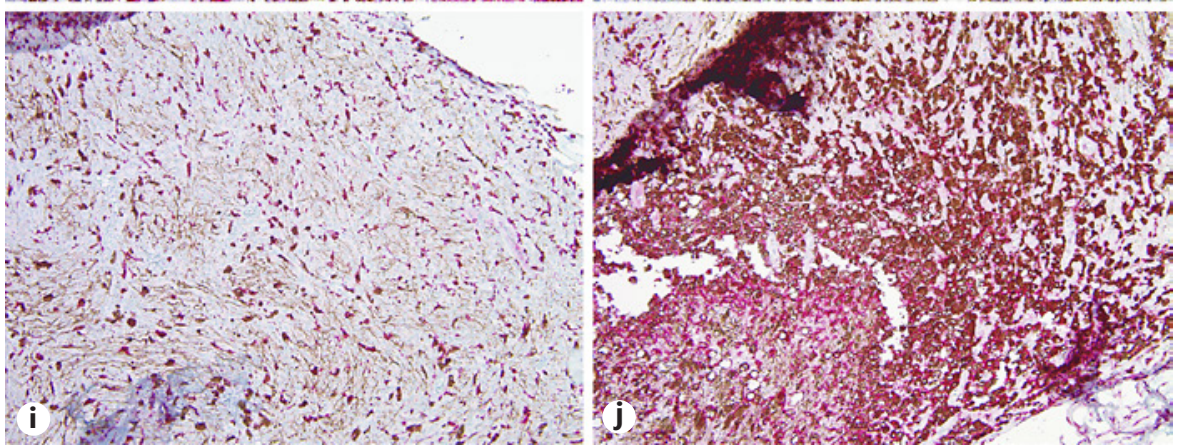
Fig. 2. EOE pattern of recurrent choroidal melanoma at low magnification (a) with an orbital nodule along the external scleral surface. High magnification image showing melanoma infiltration in transscleral emissary channel (b).

Fig. 3. Diffuse pattern of recurrent choroidal melanoma with $\mathrm{H} \& \mathrm{E}$ at low (a) and high (b) magnification. Ki-67 staining (c) shows active tumor proliferation without clear tumor zonation. HMB45 stain correlated with $\operatorname{SOX} 10(\mathbf{d}, \mathbf{e})$ showing a diffuse pattern of malignant melanoma cells. Tumor macrophages are shown with CD-163 (f).
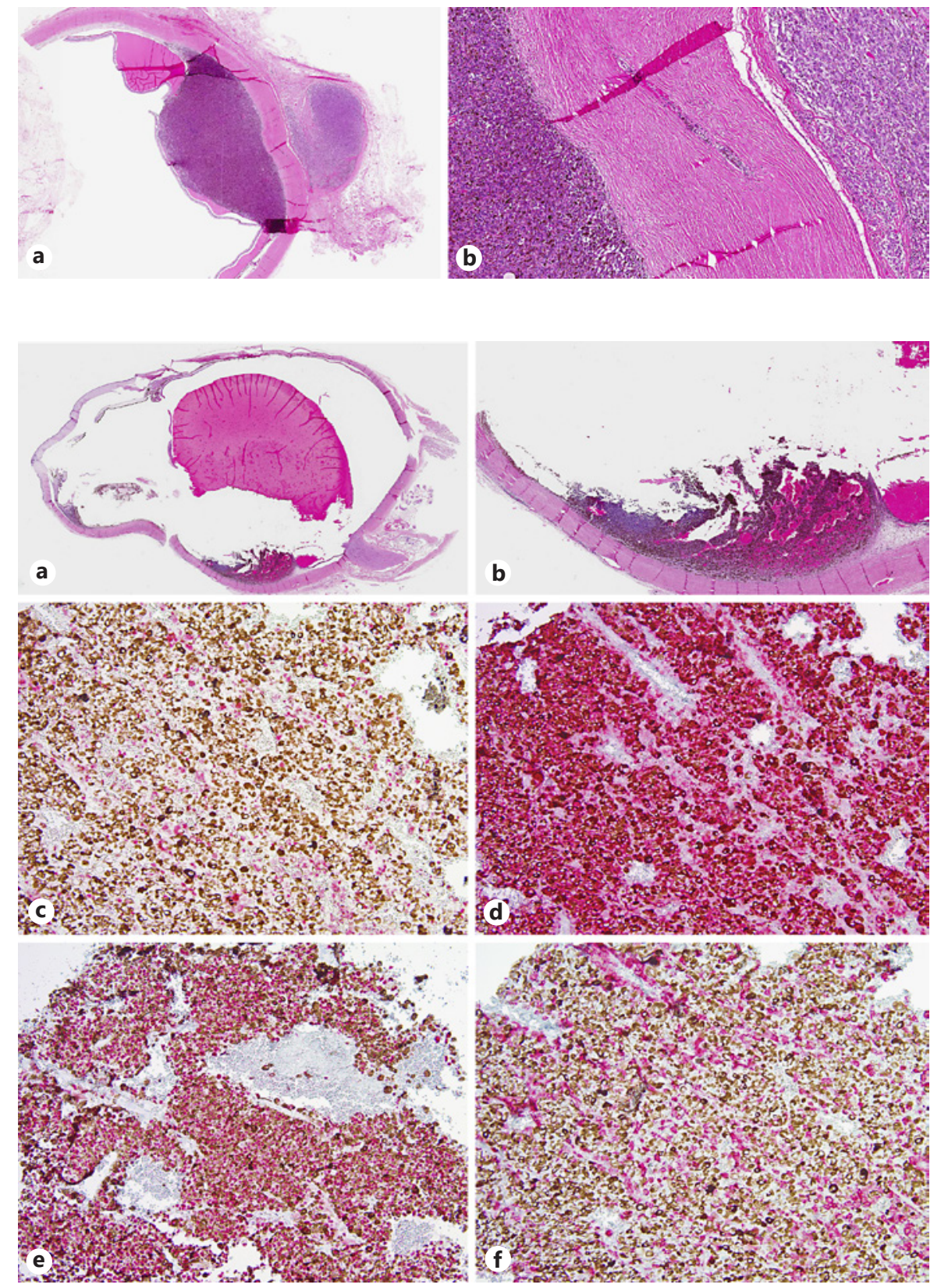

The contrasting histologic patterns of local recurrence suggest that these are possibly driven by different mechanisms of local tumor recurrence after brachytherapy (Table 4). Marginal recurrences can be explained by failure to adequately treat tumor cells along the margins of the tumor. This is especially relevant when all of our marginal recurrent cases showed tumor zonation between recurrent and nonrecurrent tumor. In a previous descriptive study, 3 cases were described to have a histological transition of malignant cells within the margin of the irradiated recurrent tumor [4]. Marginal recurrences can result from an underestimation of tumor basal diameter at initial evaluation. Additionally, inadequate tumor margin coverage at the time of plaque placement may represent a potential cause of recurrence in these cases. Therefore, marginal recurrence can be prevented with incorporation of techniques for verification of plaque placement such as intraoperative echographic confirmation of tumor margin coverage at the time of episcleral plaque placement. There is evidence to suggest that echographic confirmation of adequate plaque placement is associated with a lower risk of local recurrence $[8,9]$. Marginal re- 
Table 2. Histologic characteristics of recurrent choroidal melanoma following I-125 plaque brachytherapy

\begin{tabular}{|c|c|c|c|c|c|c|c|}
\hline 1 & Diffuse & Epithelioid & 1 & 40 & 40 & 20 & 0 \\
\hline 2 & Diffuse/central & Mixed & 0 & 60 & 30 & 10 & 0 \\
\hline 3 & Marginal & Mixed & 2 & 95 & 0 & 0 & 5 \\
\hline 4 & Marginal & Mixed & 0 & 95 & 0 & 0 & 5 \\
\hline 7 & EOE & Mixed & 3 & 95 & 0 & 5 & 0 \\
\hline
\end{tabular}

EOE, extraocular extension; HPF, high-power field.

Table 3. Immunohistochemical characterization of recurrent choroidal melanoma following I-125 plaque brachytherapy

\begin{tabular}{|c|c|c|c|c|c|c|c|c|c|c|}
\hline 1 & Diffuse & 32 & 33 & +2 & +2 & +2 & +2 & +2 & +2 & No \\
\hline 2 & Diffuse/central & 2.3 & 0.7 & +1 & +1 & +2 & +2 & +2 & +2 & No \\
\hline 3 & Marginal & 25 & 21 & +1 & +2 & +1 & +1 & +2 & +1 & Yes \\
\hline 6 & EOE & 11 & 4.2 & 0 & +1 & +3 & +1 & +2 & +1 & Yes \\
\hline 7 & EOE & 26 & 11 & +1 & +2 & +3 & +2 & +3 & +2 & Yes \\
\hline
\end{tabular}

EOE, extraocular extension; n/a, not applicable.

Table 4. Potential mechanisms of recurrence following brachytherapy in choroidal melanoma

\begin{tabular}{lll}
\hline $\begin{array}{l}\text { Histologic recurrence } \\
\text { pattern }\end{array}$ & Mechanism of recurrence & Potential preventive strategy \\
\hline Marginal & Poor tumor margin coverage & $\begin{array}{l}\text { Wider tumor margin coverage echographic } \\
\text { confirmation of tumor margin coverage }\end{array}$ \\
\hline EOE & $\begin{array}{l}\text { Pretreatment transscleral } \\
\text { emissary channel tumor } \\
\text { invasion }\end{array}$ & $\begin{array}{l}\text { Wider tumor margin coverage echographic } \\
\text { confirmation of tumor margin coverage }\end{array}$ \\
\hline Diffuse & Primary radioresistance & Tumor radiosensitization \\
\hline \multicolumn{2}{c}{ EOE, extraocular extension. } & \\
\hline
\end{tabular}

currences may be treated with a second plaque, TTT, or with enucleation depending upon the location of the tumor and potential for vision [2].

EOE cases showed microinvasion of transscleral emissary channels extending beyond the clinically visible tumor margins. Nonnecrotic melanoma tumor cells were observed in the transscleral channels extending to the extraocular space, even when both cases underwent echographic confirmation of tumor margin coverage at the time of plaque brachytherapy. Therefore, these ex- 
tensions may be missed by clinical examination and ultrasonography and are therefore difficult to determine in the pretreatment evaluation. Potential prevention strategies may include adopting tumor coverage safety margins wider than the $2 \mathrm{~mm}$ standard as established by the COMS and recommended by the Ophthalmic Oncology Task Force of the American Brachytherapy Society $[10,11]$. However, changing the guidelines for the treatment of choroidal melanoma with episcleral plaque brachytherapy may be impractical due to the proportionally low incidence of EOE [2]. The risk of using safety margins wider than the $2 \mathrm{~mm}$ standard may therefore cause unwanted ocular toxicity and longterm visually threatening complications. At this time, enucleation is recommended as definitive treatment of cases with EOE.

A third mechanism of recurrence, the diffuse type, appears to be a distinct clinicopathologic entity. Diffuse recurrence may represent a primary form of radioresistance. A clinical study of repeat episcleral plaque brachytherapy for locally recurrent posterior uveal melanoma showed that all marginal recurrences responded after the second plaque treatment, while all cases that did not respond to the second plaque were of the diffuse type [3]. The authors suggested that diffusely recurrent tumors were radioresistant due to relative hypoxia from a more thoroughly radiated vascular bed. In addition, our group reported 3 cases of diffuse or central recurrences successfully treated with additional $85 \mathrm{~Gy}$ of radiation delivered via repeat episcleral plaque application [2]. Mechanisms of radioresistance in uveal melanoma have been previously explored in both in vitro and in vivo studies [1214]. Uveal melanoma cell lines exhibit varying degrees of radiosensitivity, with some showing a higher capacity to repair sublethal DNA damage [15]. Diffuse recurrences may therefore represent a melanoma subtype with a different biological profile that makes it resistant to radiation. However, defining a preventive strategy for these tumors in the absence of clinical markers of radioresistance is a challenging task. A potential management strategy could be through tumor radiosensitization before repeat plaque brachytherapy. Thus, establishing an animal model of uveal melanoma would provide a platform to test novel drug therapies and their potential radiosensitizing effects on the radioresistant subtypes of uveal melanoma cells [16].

In conclusion, based upon IHC analysis, our observations provide a correlation between histopathologic and clinical patterns of local recurrence of choroidal melanoma after brachytherapy. The mechanism of the marginal recurrence may be explained by either imprecise tumor margin coverage or underestimation of tumor dimensions. EOE may represent a subtype of marginal recurrence. Diffuse recurrences may represent a distinct clinicopathologic entity of primary radioresistance in choroidal melanoma.

\section{Statement of Ethics}

The authors have no ethical conflicts to disclose.

\section{Disclosure Statement}

The authors have no conflicts of interest to declare.

\section{Funding Sources}

None.

\section{References}

1 Harbour JW, Char DH, Kroll S, Quivey JM, Castro J. Metastatic risk for distinct patterns of postirradiation local recurrence of posterior uveal melanoma. Ophthalmology. 1997 Nov;104(11):1785-92; discussion 1792-3.

2 Bellerive C, Aziz HA, Bena J, Wilkinson A, Suh JH, Plesec T, et al. Local Failure After Episcleral Brachytherapy for Posterior Uveal Melanoma: Patterns, Risk Factors, and Management. Am J Ophthalmol. 2017 May;177: 9-16.

3 King B, Morales-Tirado VM, Wynn HG, Gao BT, Ballo MT, Wilson MW. Repeat Episcleral Plaque Brachytherapy: Clinical Outcomes in Patients Treated for Locally Recurrent Poste- rior Uveal Melanoma. Am J Ophthalmol. 2017 Apr;176:40-5.

4 Shields CL, Shields JA, Karlsson U, Menduke $\mathrm{H}$, Brady LW. Enucleation after plaque radiotherapy for posterior uveal melanoma. Histopathologic findings. Ophthalmology. 1990 Dec;97(12):1665-70.

5 Histopathologic characteristics of uveal melanomas in eyes enucleated from the Collaborative Ocular Melanoma Study. COMS report no. 6. Am J Ophthalmol. 1998 Jun;125(6):745-66.

6 Avery RB, Diener-West M, Reynolds SM, Grossniklaus HE, Green WR, Albert DM. Histopathologic characteristics of choroidal melanoma in eyes enucleated after iodine 125 brachytherapy in the collaborative ocular melanoma study. Arch Ophthalmol. 2008 Feb;126(2):207-12

7 Pe'er J, Stefani FH, Seregard S, Kivela T, Lommatzsch P, Prause JU, et al. Cell proliferation activity in posterior uveal melanoma after $\mathrm{Ru}$ 106 brachytherapy: an EORTC ocular oncology group study. Br J Ophthalmol. 2001 Oct; 85(10):1208-12.

8 Tabandeh H, Chaudhry NA, Murray TG, Ehlies F, Hughes R, Scott IU, et al. Intraoperative echographic localization of iodine-125 episcleral plaque for brachytherapy of choroidal melanoma. Am J Ophthalmol. $2000 \mathrm{Feb}$; 129(2):199-204. 
9 Aziz HA, Al Zahrani YA, Bena J, Lorek B, Wilkinson A, Suh J, et al. Episcleral brachytherapy of uveal melanoma: role of intraoperative echographic confirmation. Br J Ophthalmol. 2017 Jun;101(6):747-51.

10 Simpson ER, Gallie B, Laperrierre N, Beiki-Ardakani A, Kivelä T, Raivio V, et al.; American Brachytherapy Society - Ophthalmic Oncology Task Force. Electronic address: paulfinger@ eyecancer.com; ABS - OOTF Committee. The American Brachytherapy Society consensus guidelines for plaque brachytherapy of uveal melanoma and retinoblastoma. Brachytherapy. 2014 Jan-Feb;13(1):1-14.

11 Jampol LM, Moy CS, Murray TG, Reynolds SM, Albert DM, Schachat AP, et al.; Collab- orative Ocular Melanoma Study Group (COMS Group). The COMS randomized trial of iodine 125 brachytherapy for choroidal melanoma: IV. Local treatment failure and enucleation in the first 5 years after brachytherapy. COMS report no. 19. Ophthalmology. 2002 Dec;109(12):2197-206.

12 Fernandes BF, Marshall JC, Di Cesare S, Logan P, Maloney S, Burnier MN Jr. Amfenac increases the radiosensitivity of uveal melanoma cell lines. Eye (Lond). 2008 May;22(5): 701-6.

13 Nagane M, Kanai E, Shibata Y, Shimizu T, Yoshioka C, Maruo T, et al. Sulfasalazine, an inhibitor of the cystine-glutamate antiporter, reduces DNA damage repair and en- hances radiosensitivity in murine $\mathrm{B} 16 \mathrm{~F} 10$ melanoma. PLoS One. 2018 Apr; 13(4): e0195151.

14 Sun Y, Tran BN, Worley LA, Delston RB, Harbour JW. Functional analysis of the p53 pathway in response to ionizing radiation in uveal melanoma. Invest Ophthalmol Vis Sci. 2005 May;46(5):1561-4.

15 van den Aardweg GJ, Naus NC, Verhoeven AC, de Klein A, Luyten GP. Cellular radiosensitivity of primary and metastatic human uveal melanoma cell lines. Invest Ophthalmol Vis Sci. 2002 Aug;43(8):2561-5.

16 Cao J, Jager MJ. Animal Eye Models for Uveal Melanoma. Ocul Oncol Pathol. 2015 Apr; $1(3): 141-50$. 